\title{
Produtividade da cana-de-açúcar e definição de zonas específicas de manejo do solo
}

\section{Productivity of the sugarcane and definition of specific zones of soil management}

\author{
Flávio Carlos Dalchiavon ${ }^{1 *}$; Morel de Passos e Carvalho ${ }^{2}$; \\ Flávio Gomes de Andrade ${ }^{3}$; Rafael Montanari²; Marcelo Andreotti ${ }^{2}$
}

\begin{abstract}
Resumo
A correta intervenção espacial na administração da lavoura, decorrente das zonas específicas de manejo do solo, pode aumentar sua produtividade e a lucratividade agrícola. No ano de 2010, no município

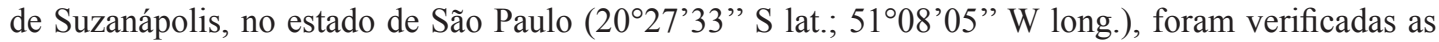
correlações (espaciais e lineares), entre atributos da cana-de-açúcar e alguns atributos químicos de um Argissolo Vermelho distrófico, visando encontrar aqueles que melhor se correlacionassem com a produtividade da cultura. Para tanto, instalou-se a malha para a coleta de dados do solo e da planta, com 118 pontos amostrais, num talhão de 10,5 ha com a cana-de-açúcar de terceiro corte. A produtividade de cana-de-açúcar (PRO) representou o atributo da planta, enquanto que os do solo foram: $\mathrm{K}^{+}, \mathrm{Ca}^{+2}, \mathrm{Mg}^{+2}$ e matéria orgânica, nas profundidades de 0-0,20 m e 0,20-0,40 m. Estabeleceram-se correlações entre a PRO e os atributos do solo. Foram modelados semivariogramas para todos os atributos, obtendo-se as respectivas krigagens e validações cruzadas. Também foram estabelecidas as cokrigagens entre a PRO e os atributos do solo. Os teores de matéria orgânica do solo, por evidenciarem substanciais correlações, Sperman's Rho e espaciais, com a produtividade de colmos de cana-de-açúcar, são indicadores de duas zonas específicas de manejo do solo fortemente associadas à produtividade da cana-de-açúcar. Nelas, tal produtividade varia entre 75,8-94,7 e 101,0-119,9 $\mathrm{t} \mathrm{ha}^{-1}$ quando respectivamente os teores de matéria orgânica forem de 12,7-14,0 e 14,5-15,8 $\mathrm{g} \mathrm{dm}^{-3}(0-0,20 \mathrm{~m})$ e $11,8-12,8$ e 13,1-14,0 $\mathrm{g} \mathrm{dm}^{-3}(0,20-0,40 \mathrm{~m})$. Palavras-chave: Geoestatística, manejo e conservação do solo, Saccharum spp., variabilidade espacial
\end{abstract}

\footnotetext{
Abstract

The correct spatial intervention in the administration of the plantation, arising from specific areas of soil mapping, can increase your productivity as well as profitability and yields in agriculture. The spatial and Pearson's relationships between sugarcane attributes and chemical attributes of a Typic Tropustalf were studied in the growing season of 2010, in Suzanápolis, State of São Paulo, Brazil (20²7'33" $\mathrm{S}$ lat.; $51^{\circ} 08^{\prime} 05^{\prime} \mathrm{W}$ long.), in order to obtain the attributes that had the best sugarcane productivity relationship. To this end, a geostatistical grid containing 118 sample points was installed for soil and plant data collection in an area of 10.5 ha with the third crop cut. The productivity of sugarcane (PRO) represented the attribute of the plant, while the attributes of the soil were: $\mathrm{K}^{+}, \mathrm{Ca}^{+2}, \mathrm{Mg}^{+2}$ and organic matter at depths of $0-0.20 \mathrm{~m}$ and $0.20-0.40 \mathrm{~m}$. Relationships were calculated between the PRO and the attributes of the soil. Semivariograms were adjusted for all attributes, obtaining the respective krigings

1 Prof. Efetivo do Instituto Federal de Mato Grosso, Campus Campo Novo do Parecis, Dept ${ }^{\circ}$ de Agronomia, Rodovia MT 235, Km 12, Zona Rural, C P 100, CEP: 78 360-000, Campo Novo do Parecis, MT. E-mail: flavio.dalchiavon@enp.ifmt.edu.br

2 Profs. do Dept ${ }^{\circ}$ de Fitossanidade, Engenharia Rural e Solos, Faculdade de Engenharia de Ilha Solteira, UNESP, Ilha Solteira, SP. E-mail: morel@agr.feis.unesp.br; montanari@agr.feis.unesp.br; dreotti@agr.feis.unesp.br

${ }^{3}$ Eng $^{\circ}$ Agr ${ }^{\circ}$, UNESP, Ilha Solteira, SP. E-mail: semogoivalf@hotmail.com

* Autor para correspondência
} 
and the cross-validations. It was also made the cokrigings between the PRO and the soil attributes. The levels of the soil organic matter, for their evident substantial correlations, Sperman's Rho and spatial, with the productivity of sugarcane, are indicators of two specific areas of soil management strongly associated with the productivity of sugarcane. In such zones this productivity varies between 75.8-94.7 $\mathrm{t} \mathrm{ha}^{-1}$ and 101.0-119.9 $\mathrm{t} \mathrm{ha}^{-1}$, when the levels of organic matter respectively are $12.7-14.5 \mathrm{~g} \mathrm{dm}^{-3}(0-0.20$ $\mathrm{m})$ and $11.8-12.8 \mathrm{~g} \mathrm{dm}^{-3}(0.20-0.40 \mathrm{~m})$.

Key words: Geostatistical, soil management and conservation, Saccharum spp., spatial variability

\section{Introdução}

No Brasil, a cana-de-açúcar (Sacchharum officinarum L.) é de inquestionável importância socioeconômica. Constitui-se na principal matériaprima para a produção do etanol combustível automotor, além da sua destinação para a produção do açúcar, um dos principais produtos de exportação nacional. Na safra nacional 2010/11, foram processadas 623,9 milhões de toneladas de colmos. Desse montante, o estado de São Paulo participou com 57,8\%, numa área de 4,36 milhões de hectares, e com a produtividade média de $83,0 \mathrm{t}$ ha $^{-1}$ (CONAB, 2011).

A agricultura de precisão determina o exato manejo da lavoura tendo como base o mapeamento de zonas específicas de manejo do solo. Seus maiores benefícios são a redução do custo de produção devido ao menor gasto com insumos, oriundo da aplicação com taxa variada, e o aumento da produtividade agrícola (DALCHIAVON et al., 2012). A análise da variabilidade do solo por meio da geoestatística possibilita o ajuste do semivariograma para dados georreferenciados que apresentem dependência espacial. Contudo, havendo afinidade entre as dependências espaciais de dois atributos quaisquer, modelada pelo semivariograma cruzado, pode-se obter o mapa de cokrigagem para o atributo principal, de difícil obtenção e de maior interesse, em função do atributo secundário, normalmente de fácil obtenção (MOLIN et al., 2007; MONTANARI et al., 2010). Poder-se-ia, então, a partir do atributo secundário, obter as zonas específicas de manejo do solo para o atributo primário.
Nos últimos anos alguns trabalhos têm sido desenvolvidos com o propósito de pesquisar a relação espacial entre os atributos do solo (secundários) e a produtividade das culturas (principal). Dentre eles, são referidos os de Martins et al. (2009), Lima et al. (2010) e Braga (2011), respectivamente com as culturas do feijoeiro, eucalipto e da cana-de-açúcar. Assim, o objetivo do trabalho foi caracterizar as zonas específicas de manejo do solo, empregando-se correlações entre a produtividade de cana-de-açúcar e atributos químicos de um Argissolo Vermelho distrófico de Suzanápolis (SP), no sentido de indicar aquele que mais eficientemente esteja relacionado com o aumento da produtividade.

\section{Material e Métodos}

O estudo foi desenvolvido em 2010, em Suzanápolis (SP, Brasil), latitude 20²7'33" $\mathrm{S}$, longitude $51^{\circ} 08^{\prime} 05^{\prime}$ 'W. De acordo com a classificação de Köppen, o clima é $\mathrm{A}_{\mathrm{w}}$, tropical úmido com estação chuvosa no verão e seca no inverno. Na Figura 1 estão apresentadas as precipitações e temperaturas médias durante o período experimental, cujos valores médios foram de $1456 \mathrm{~mm}$ ano $^{-1}$, temperatura máxima, média e mínima de 30,8; 24,5 e $19,4{ }^{\circ} \mathrm{C}$, respectivamente. O solo foi classificado como Argissolo Vermelho distrófico abrúptico textura média/argilosa, A moderado (EMBRAPA, 2006), com declive homogêneo de $0,065 \mathrm{~m} \mathrm{~m}^{-1}$. 
Figura 1. Precipitação pluvial e médias térmicas obtidas durante o período experimental (maio/2008 a maio/2010).

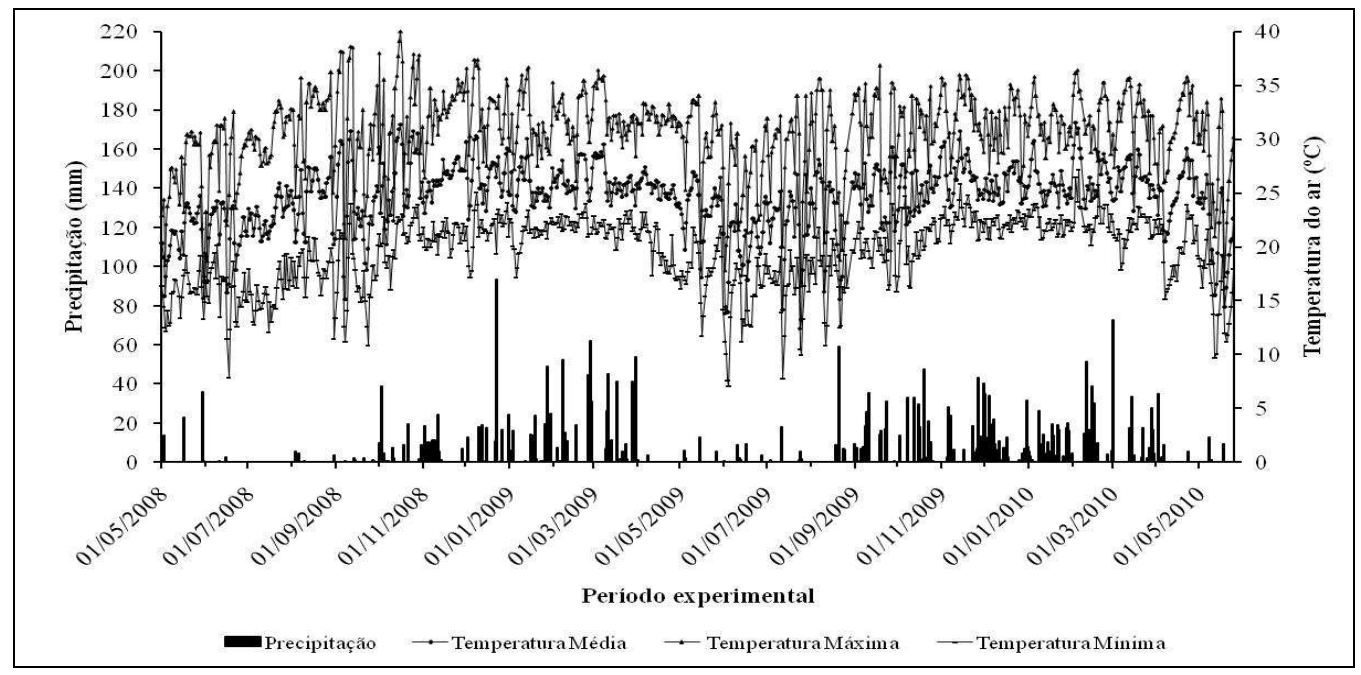

Fonte: Elaboração dos autores. (dados fornecidos pela Usina Vale do Paraná Açúcar e Álcool S/A).

Instalou-se em 25/05/2010, num talhão de 10,5 ha com cana-de-açúcar (variedade RB85 5453) plantada em 23/06/2006 no espaçamento de 1,5 m. A malha para coleta de dados foi constituída de 100 pontos amostrais, espaçados em $33,94 \mathrm{~m}$, acrescida de seis malhas de refinamento, alocadas com o objetivo de detectar alcances da dependência espacial para espaçamentos menores que $30 \mathrm{~m}$, mais 18 pontos amostrais, espaçados em 16,97 m (Figura 2). Assim, o total de pontos amostrais foi de 118 , onde foram coletados os atributos de solo e planta. Os pontos amostrais foram georreferenciados com aparelho GPS geodésico Sokkia Straus L1/L2, na projeção UTM e datum SIRGAS-2000, zona 22K. As direções dos eixos cartesianos da malha de dados foram estabelecidas de modo que a longitude correspondeu ao eixo $\mathrm{X}$ e a latitude, ao eixo $\mathrm{Y}$.

Os atributos químicos do solo, determinados nas profundidades de 0-0,20 $\mathrm{m}$ e 0,20-0,40 m, foram: teores de potássio $(\mathrm{K})$, de cálcio $(\mathrm{Ca})$, de magnésio $(\mathrm{Mg})$, em $\mathrm{mmol}_{\mathrm{c}} \mathrm{dm}^{-3}$, e de matéria orgânica do solo (MO) em $\mathrm{g} \mathrm{dm}^{-3}$, determinados conforme Raij et al. (2001). O da planta foi a produtividade de colmos (PRO) em t ha ${ }^{-1}$. A cana encontrava-se em seu terceiro corte (bisada), sendo colhida manualmente após despalha a fogo. A PRO foi obtida pela colheita das canas presentes nas duas linhas adjacentes ao ponto estaqueado. Como o espaçamento entrelinhas foi de 1,50 m, elas representaram 3,00 m. Portanto, tomando-se também 3,00 m no sentido do plantio, a área amostral de cada ponto foi de $9 \mathrm{~m}^{2}(3,0 \mathrm{~m}$ $\mathrm{x} 3,0 \mathrm{~m})$. A pesagem das canas representantes de cada ponto foi efetuada imediatamente após o corte, no campo, com balança analítica eletrônico-digital $(+/-0,02 \mathrm{~kg})$, com capacidade máxima para 50,0 kg. A transformação das pesagens, ponto por ponto, foi dada por: 
Figura 2. Esquema da malha experimental de campo.

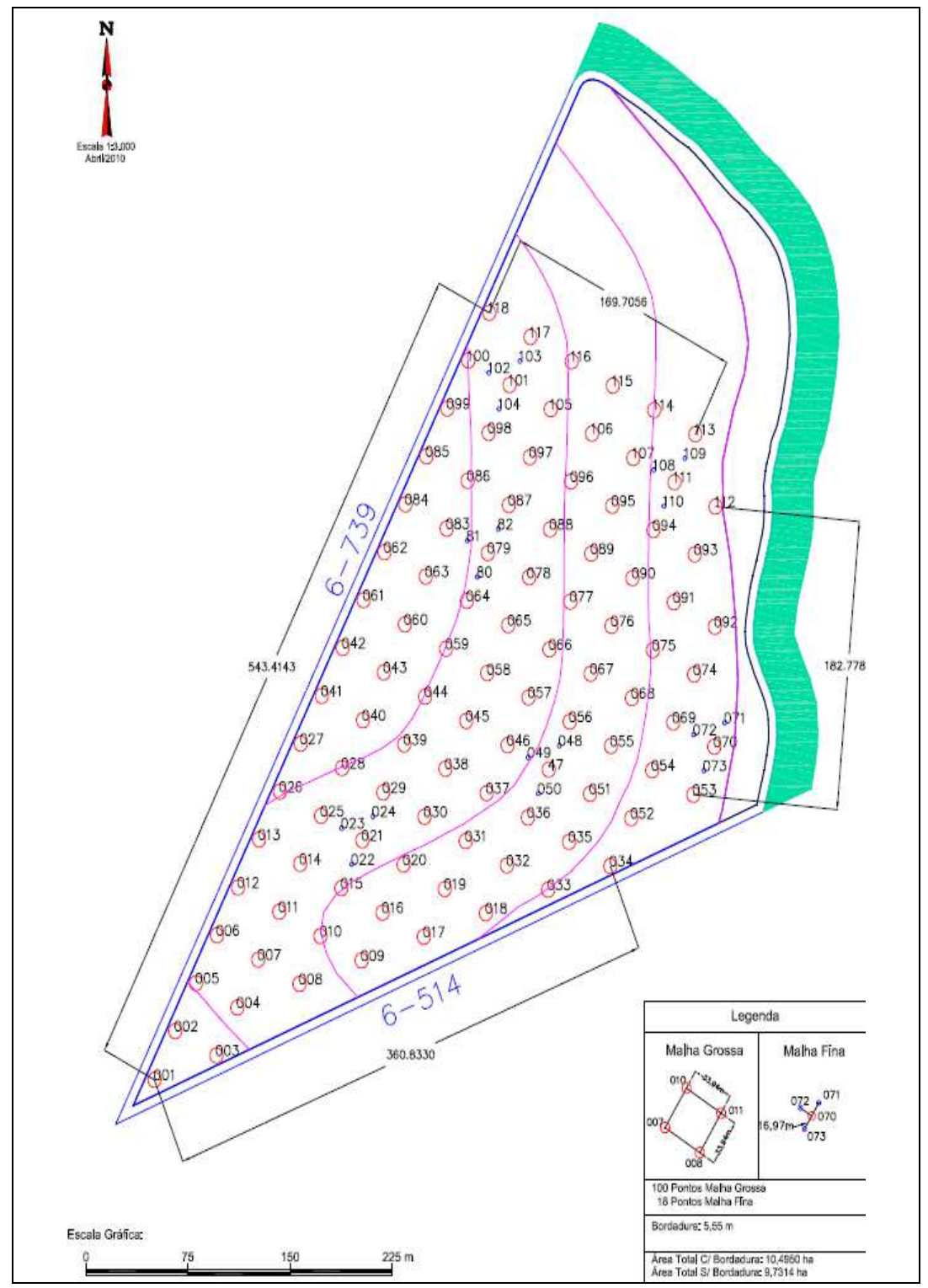

Fonte: Usina Vale do Paraná Açúcar e Álcool S/A.

PRO $=1111 . m$

onde: PRO é a produtividade de colmos $\left(\mathrm{t} \mathrm{ha}^{-1}\right)$ e $\mathbf{m}$ a massa dos colmos contidos na área amostral de 9 $\mathrm{m}^{2}(\mathrm{~kg})$.

A análise estatística foi efetuada com o software Statistical Analysis System - SAS (SCHLOTZHAVER; LITTLEL, 1997) e a planilha Excel, seguindo os procedimentos de Dalchiavon et al. (2011a, 2001b). Foi efetuada a análise descritiva dos atributos, calculando-se a média, a mediana, os valores mínimos e máximos, o desvio padrão, o coeficiente de variação, de curtose, de assimetria, e a análise da distribuição de frequência pelo teste de Shapiro e Wilk (1965) a 5\%. Foi montada a matriz de correlação entre todos os atributos pesquisados, contendo todas as combinações pareadas possíveis. Objetivou-se detectar a existência de correlações significativas entre os atributos para efetuar regressões da $\mathrm{PRO}$ em função dos atributos do 
solo, no intuito de rastrear a existência de um deles, o qual pudesse funcionar como indicador de qualidade, quando o objetivo fosse o de aumentar a produtividade da cana-de-açúcar.

A análise geoestatística foi feita com o auxílio do Software Gamma Design 7.0 (GS+, 2004), seguindo os procedimentos de Dalchiavon et al. (2012). Para cada atributo foi analisada a dependência espacial, pelo cálculo do semivariograma, onde os ajustes foram efetuados prioritariamente pela seleção inicial de: (a) menor soma dos quadrados dos desvios (RSS); (b) maior coeficiente de determinação $\left(\mathrm{r}^{2}\right)$; e (c) maior avaliador da dependência espacial (ADE), pela equação contida abaixo:

$\mathrm{ADE}=[\mathrm{C} /(\mathrm{C}+\mathrm{Co})] .100$

em que $\mathrm{ADE}$ é o avaliador da dependência espacial; C é a variância estrutural; e C + Co é o patamar. A interpretação para o ADE foi conforme sugestão de Dalchiavon et al. (2012), sendo a seguinte: a) $\mathrm{ADE}<20 \%=$ variável espacial de muito baixa dependência (MB); b) $20 \% \leq \mathrm{ADE}<$ $40 \%=$ baixa dependência (BA); c) $40 \% \leq \mathrm{ADE}$ $<60 \%=$ média dependência (ME); d) $60 \% \leq$ $\mathrm{ADE}<80 \%=$ alta dependência (AL) e e) $80 \% \leq$ $\mathrm{ADE}<100 \%=$ muito alta dependência (MA). Na indefinição de estacionaridade para algum atributo (ATR), extraia-se a tendência dos seus dados pelo método da regressão polinomial (DAVIS, 1986), trabalhando-se, desta forma, com os resíduos dos seus dados. Assim, eles ficaram precedidos do símbolo \# quando referidos na tabela da análise semivariográfica e da validação cruzada (\#ATR). Já quando referidos no mapa de krigagem e/ou cokrigagem, ficaram precedidos por $£$ (£ATR). Efetuaram-se cokrigagens entre a PRO e todos os demais atributos que apresentaram dependência espacial.

\section{Resultados e Discussão}

A PRO de colmos de cana-de-açúcar $\left(89,5 \mathrm{tha}^{-1}\right)$ indicada na Tabela 1 , foi inferior às $100 \mathrm{t} \mathrm{ha}^{-1}$ obtidas por Watanabe, Fioretto e Hermann (2004) para cana de terceiro corte, variedade RB85-5536, cultivada num Latossolo Vermelho distroférrico. Entretanto, foi 13,7\% superior à obtida por Souza et al. (2008), variedade SP80-1816, em um Latossolo VermelhoAmarelo. Por outro lado, a PRO do presente estudo é semelhante à obtida por Braga (2011), para cana de segundo corte (variedade SP 79 1011), cultivada em um Argissolo Vermelho eutrófico. Contudo, era de se esperar uma PRO mais elevada, por se tratar de cana com 24 meses de ciclo (bisada).

A distribuição de frequência normal, representante típica dos dados da planta, é a ideal para um estudo estatístico (análise de regressão e/ou geoestatística), ainda que na geoestatística, mais importante que a normalidade dos dados é a ocorrência ou não do efeito proporcional em que a média e a variância dos dados não sejam constantes na área de estudo (DALCHIAVON et al., 2012). Não sendo assim, busca-se a normalidade pela transformação logarítmica (MOLIN et al., 2007). A PRO apresentou distribuição de frequência normal, com curtose e assimetria respectivamente de 0,075 e 0,143 (Tabela 1), assim como o Ca1 e o Ca2 também revelaram normalidade nos dados, ficando plenamente de acordo com Braga (2011), que verificou distribuição de frequência normal para a PRO da cana-soca num Argissolo Vermelho eutrófico, indicando que as medidas de tendência central não refletem valores atípicos na distribuição. Por outro lado, distribuição de frequência do tipo lognormal $(\mathrm{K})$, como já havia sido constatada por Montanari et al. (2008) em Argissolo, e indeterminada ( $\mathrm{gg}$ e $\mathrm{MO}$ ) também foram observadas no presente estudo. 
Tabela 1. Análise descritiva da produtividade da cana-de-açúcar e de alguns atributos químicos de um Argissolo Vermelho distrófico de Suzanápolis (SP).

\begin{tabular}{|c|c|c|c|c|c|c|c|c|c|c|}
\hline \multirow{2}{*}{ Atributo (a) } & \multirow{2}{*}{ Média } & \multirow{2}{*}{ Mediana } & \multicolumn{2}{|c|}{ Valor } & \multirow{2}{*}{$\begin{array}{l}\text { Desvio } \\
\text { Padrão }\end{array}$} & \multicolumn{3}{|c|}{ Coeficiente } & \multicolumn{2}{|c|}{$\begin{array}{l}\text { Probabilidade } \\
\text { do teste (b) }\end{array}$} \\
\hline & & & Mínimo & Máximo & & $\begin{array}{c}\text { Variação } \\
(\%)\end{array}$ & Curtose & Assimetria & $\operatorname{Pr}<\mathbf{w}$ & DF \\
\hline \multicolumn{11}{|c|}{ Atributo da planta } \\
\hline $\operatorname{PRO}\left(\mathrm{t} \mathrm{ha} \mathbf{h}^{-1}\right)$ & 89,5 & 90,3 & 42,0 & 153,0 & 20,6 & 23,0 & 0,075 & 0,143 & 0,386 & NO \\
\hline \multicolumn{11}{|c|}{ Atributos do solo } \\
\hline $\mathrm{K} 1\left(\mathrm{mmol}_{\mathrm{c}} \mathbf{d m}^{-3}\right)$ & 2,0 & 1,9 & 0,7 & 4,2 & 0,8 & 38,8 & $-0,493$ & $-0,304$ & 0,088 & $\mathrm{LN}$ \\
\hline $\mathrm{K} 2\left(\mathrm{mmol}_{\mathrm{c}} \mathrm{dm}^{-3}\right)$ & 1,0 & 0,8 & 0,3 & 3,2 & 0,6 & 53,8 & $-0,527$ & 0,173 & 0,065 & $\mathrm{LN}$ \\
\hline $\mathrm{Ca} 1\left(\mathrm{mmol}_{\mathrm{c}} \mathrm{dm}^{-3}\right)$ & 9,2 & 9,0 & 3,0 & 16,0 & 2,4 & 26,0 & 0,037 & 0,051 & 0,083 & NO \\
\hline $\mathrm{Ca} 2\left(\mathrm{mmol}_{\mathrm{c}} \mathrm{dm}^{-3}\right)$ & 10,8 & 11,0 & 4,0 & 18,0 & 2,8 & 26,1 & $-0,182$ & 0,199 & 0,072 & NO \\
\hline $\operatorname{Mg} 1\left(\operatorname{mmol}_{\mathrm{c}} \mathrm{dm}^{-3}\right)$ & 5,3 & 5,0 & 1,0 & 17,0 & 2,1 & 38,1 & 8,012 & 1,601 & $10^{-4}$ & $\mathrm{IN}$ \\
\hline $\operatorname{Mg} 2\left(\operatorname{mmol}_{\mathrm{c}} \mathrm{dm}^{-3}\right)$ & 6,9 & 7,0 & 3,0 & 13,0 & 2,3 & 32,7 & $-0,180$ & 0,444 & 0,002 & IN \\
\hline MO1 (g dm $\left.{ }^{-3}\right)$ & 14,5 & 14,0 & 11,0 & 18,0 & 1,6 & 11,2 & $-0,601$ & 0,292 & $10^{-4}$ & IN \\
\hline $\operatorname{MO2}\left(\mathrm{g} \mathrm{dm}^{-3}\right)$ & 13,5 & 13,0 & 10,0 & 19,0 & 1,7 & 2,9 & 0,984 & 0,633 & $10^{-4}$ & IN \\
\hline
\end{tabular}

(a) $\mathrm{PRO}, \mathrm{K}, \mathrm{Ca}, \mathrm{Mg}$, MO são respectivamente a produtividade de colmos de cana-de-açúcar por hectare, teores de potássio, de cálcio, de magnésio e de matéria orgânica do solo; os índices 1 e 2 referem-se respectivamente às profundidades de solo 0-0,20 e 0,20-0,40 m; ${ }^{\text {(b) }} \mathrm{DF}=$ distribuição de frequência, sendo NO, LN e IN respectivamente do tipo normal, lognormal e indeterminada.

Fonte: Elaboração dos autores.

No estudo das correlações, como a MO1 resultou distribuição de frequência indeterminada, o coeficiente de correlação de Sperman's Rho entre PRO x MO1 foi der $=0,294 * *$. As demais correlações significativas entre os atributos estão expressas na Tabela 2. A MO1 foi o atributo de interesse do solo significativo com a PRO, indicando relação de independência direta entre eles, corroborando os dados obtidos por Souza et al. (2008) e Braga (2011). Apesar de significativo, o coeficiente de correlação apresenta baixa magnitude basicamente devido ao elevado número de observações $(\mathrm{n}=118)$. Deste modo, a principal equação ajustada foi:

$$
\mathrm{PRO}=3,76^{* *} . \mathrm{MO} 1+35,17 \ldots \ldots . .(\mathrm{r}=0,294 * *) \ldots
$$

A equação 3 indicou influência linear direta do teor de matéria orgânica do solo sobre a produtividade de cana-de-açúcar. Assim, para uma variação compreendida entre os teores de 11 e 18 $\mathrm{g} \mathrm{dm}^{-3}$ de MO1 (Tabela 1), estima-se que a PRO passará de 76,5 para 102,9 t ha-1 (Equação 3), assim como para o teor mediano de MO1 igual a $14 \mathrm{~g} \mathrm{dm}^{-3}$ a PRO será de $87,8 \mathrm{t} \mathrm{ha}^{-1}$. Tal equação demonstra o quão importante é a adoção de práticas agronômicas que visem elevar os teores de matéria orgânica no solo, uma vez que este atributo químico evidenciou aumentar significativamente a produtividade de colmos de cana-de-açúcar. Salienta-se ainda que a referida equação ficou plenamente de acordo com a equação de Vitti et al. (2008) e Carvalho et al. (2010), que também encontraram relação linear positiva entre a PRO de cana e a MO, ratificando a relevância da MO no âmbito do manejo e conservação do solo por influenciar substancialmente suas propriedades químicas, físicas e biológicas, com implicação direta na produtividade vegetal. 
Tabela 2. Matriz de correlação entre a produtividade da cana-de-açúcar e alguns atributos químicos de um Argissolo Vermelho distrófico de Suzanápolis (SP).

\begin{tabular}{|c|c|c|c|c|c|c|c|c|}
\hline \multirow{2}{*}{ Atributos $^{(a)}$} & \multicolumn{8}{|c|}{ Coeficiente de correlação ${ }^{(b)}$} \\
\hline & PRO & K1 & K2 & Ca1 & Ca2 & Mg1 & Mg2 & MO1 \\
\hline K1 & 0,048 & & & & & & & \\
\hline $\mathbf{K 2}$ & $-0,094$ & $0,631 * *$ & & & & & & \\
\hline Ca1 & 0,073 & 0,092 & $-0,044$ & & & & & \\
\hline $\mathrm{Ca} 2$ & 0,123 & 0,008 & $-0,192 *$ & $0,608 * *$ & & & & \\
\hline Mg1 & $-0,225^{*}$ & $0,199 *$ & 0,103 & $0,625 * *$ & $0,277 * *$ & & & \\
\hline Mg2 & $-0,134$ & 0,124 & $-0,047$ & $0,427 * *$ & $0,623 * *$ & $0,614 * *$ & & \\
\hline MO1 & $0,294 * *$ & $-0,027$ & $-0,113$ & $0,271 * *$ & $0,307 * *$ & 0,057 & 0,138 & \\
\hline MO2 & 0,160 & $-0,054$ & $-0,102$ & 0,120 & $0,346 * *$ & $-0,062$ & 0,106 & $0,503 * *$ \\
\hline
\end{tabular}

(a) Vide Tabela 1; (b) ** significativo a $1 \%$, * significativo a $5 \%$.

Fonte: Elaboração dos autores.

A análise geoestatística (Tabela 3, Figura 3) mostrou que a \#PRO apresentou coeficiente de correlação espacial $\left(\mathrm{r}^{2}\right)$ muito alto $(0,882)$, dependência espacial (ADE) média $(50,0 \%)$ e coeficiente angular (b) da validação cruzada de 0,723, conforme classificação apresentada em Dalchiavon e Carvalho (2012). Por outro lado, os atributos do solo, com dependência espacial, apresentaram $r^{2}$ entre médio $(0,494)$ e muito alto $(0,923)$, ADE entre muito baixa $(20,0 \%)$ e média $(60,0 \%)$ e coeficientes angulares ficando entre 0,798 e 0,900 (Tabela 3, Figuras 3 e 4). Esses dados foram muito semelhantes àqueles obtidos por Chaves e Farias (2008), Montanari et al. (2010) e Souza et al. (2010), quando pesquisaram as culturas da cana-de-açúcar, do feijoeiro e da cana-de-açúcar, respectivamente, bem como atributos do solo.

Os alcances semivariográficos ficaram entre 120,0 (K1) e 278,8 m (\#PRO), indicando que para manejos específicos e localizados, os valores referenciais não deverão ser menores do que 120,0 $\mathrm{m}$ (Tabela 3), por representarem a distância dentro da qual os valores de um determinado atributo apresentam correlação espacial entre si, haja vista que, segundo Corá et al. (2004), o valor do alcance pode influenciar a qualidade da estimativa, uma vez que determina o número de valores usados na interpolação. Assim, estimativas feitas com interpolação por krigagem utilizando os maiores valores do alcance tendem a ser mais confiáveis, apresentando mapas que representam melhor a realidade.

Os semivariogramas cruzados (Tabela 3, Figura 5) apresentaram-se com $\mathrm{r}^{2}$ médio $(0,406 \mathrm{e}$ $0,580)$ para as variáveis secundárias MO2 e MO1, respectivamente, corroborando os dados obtidos por Dalchiavon (2010) e Braga (2011). Desta forma, ocorreram apreciáveis correlações espaciais diretas com a £PRO (MO1 e MO2), proporcionando a definição de zonas homogêneas de manejo, o que permite a adoção do sistema de agricultura de precisão (DALCHIAVON; CARVALHO, 2012), uma vez que a definição das referidas zonas de manejo ficou fortemente evidenciada pela interação entre a produtividade da cultura e os teores de matéria orgânica do solo (Figura 5), sendo homogêneo, portanto, o manejo para toda região tida como homogênea. 
Tabela 3. Parâmetros dos semivariogramas simples e cruzados da produtividade da cana-de-açúcar e de alguns atributos químicos de um Argissolo Vermelho distrófico de Suzanápolis (SP).

\begin{tabular}{|c|c|c|c|c|c|c|c|c|c|c|c|}
\hline \multirow{3}{*}{ Atributo $^{(a)}$} & \multicolumn{11}{|c|}{ Parâmetros do ajuste } \\
\hline & \multirow{2}{*}{ Modelo $^{(\mathbf{b})}$} & \multirow{2}{*}{$\mathrm{C}_{0}$} & \multirow{2}{*}{$\mathrm{C}_{\mathrm{o}}+\mathrm{C}$} & \multirow{2}{*}{$\begin{array}{l}A_{0} \\
(\mathbf{m})\end{array}$} & \multirow{2}{*}{$\mathbf{r}^{2}$} & \multirow{2}{*}{$\mathrm{SQR}^{(\mathrm{c})}$} & \multicolumn{2}{|c|}{$\mathbf{A D E}^{(\mathbf{d})}$} & \multicolumn{3}{|c|}{ Validação cruzada } \\
\hline & & & & & & & $\%$ & Classe & a & b & $\mathbf{r}$ \\
\hline \multicolumn{12}{|c|}{$\gamma($ h) simples - Planta } \\
\hline \#PRO & $\exp$ & $1,73.10^{2}$ & $3,48.10^{2}$ & 278,8 & 0,882 & $1,85.10^{3}$ & 50,0 & ME & 24,52 & 0,723 & 0,327 \\
\hline \multicolumn{12}{|c|}{$\gamma(h)$ simples - Solo } \\
\hline K1 & $\exp$ & $2,45.10^{-1}$ & $5,60 \cdot 10^{-1}$ & 120,0 & 0,537 & $1,88.10^{-2}$ & 56,3 & $\mathrm{ME}$ & 0,30 & 0,861 & 0,339 \\
\hline $\mathbf{K} 2$ & epp & $2,99 \cdot 10^{-1}$ & $2,99 \cdot 10^{-1}$ & - & - & - & - & - & - & - & - \\
\hline Ca1 & $\exp$ & 2,91 & 6,62 & 272,1 & 0,923 & 0,608 & 56,0 & $\mathrm{ME}$ & 0,95 & 0,900 & 0,445 \\
\hline $\mathrm{Ca} 2$ & epp & 7,74 & 7,74 & - & - & - & - & - & - & - & - \\
\hline Mg1 & gau & 2,40 & 3,00 & 170,0 & 0,494 & $3,58.10^{-1}$ & 20,0 & MB & 1,09 & 0,798 & 0,235 \\
\hline Mg2 & epp & 5,22 & 5,22 & - & - & - & - & - & - & - & - \\
\hline MO1 & $\exp$ & $9,64 \cdot 10^{-1}$ & 2,41 & 249,0 & 0,647 & $5,08.10^{-1}$ & 60,0 & $\mathrm{ME}$ & 1,99 & 0,863 & 0,437 \\
\hline MO2 & gau & 1,86 & 2,70 & 210,0 & 0,652 & $5,35 \cdot 10^{-1}$ & 31,2 & BA & 1,84 & 0,859 & 0,415 \\
\hline \multicolumn{12}{|c|}{$\gamma(h)$ cruzado entre a \#PRO $x$ atributos do Solo } \\
\hline MO1 & esf & 2,28 & 8,56 & 261,9 & 0,580 & $2,36.10^{1}$ & 73,4 & $\mathrm{AL}$ & $-6,79$ & 0,574 & 0,345 \\
\hline MO2 & esf & 3,52 & 9,32 & 287,5 & 0,406 & $4,56.10^{1}$ & 62,2 & $\mathrm{AL}$ & $-7,91$ & 0,493 & 0,311 \\
\hline
\end{tabular}

(a) Vide Tabela 1; \# trabalhado com o resíduo do atributo; ${ }^{(b)}$ exp = exponencial, epp = efeito pepita puro, gau = gaussiano e esf = esférico; ${ }^{(c)} \mathrm{SQR}=$ soma dos quadrados dos resíduos; ${ }^{(\mathrm{d})} \mathrm{ADE}=$ avaliador da dependência espacial, sendo $\mathrm{ME}=$ média, $\mathrm{MB}=$ muito baixa, $\mathrm{BA}=$ baixa e $\mathrm{AL}=$ alta.

Fonte: Elaboração dos autores. 
Figura 3. Semivariogramas simples e mapas de krigagem da produtividade da cana-de-açúcar, da MO1 e da MO2 de um Argissolo Vermelho distrófico de Suzanápolis (SP).

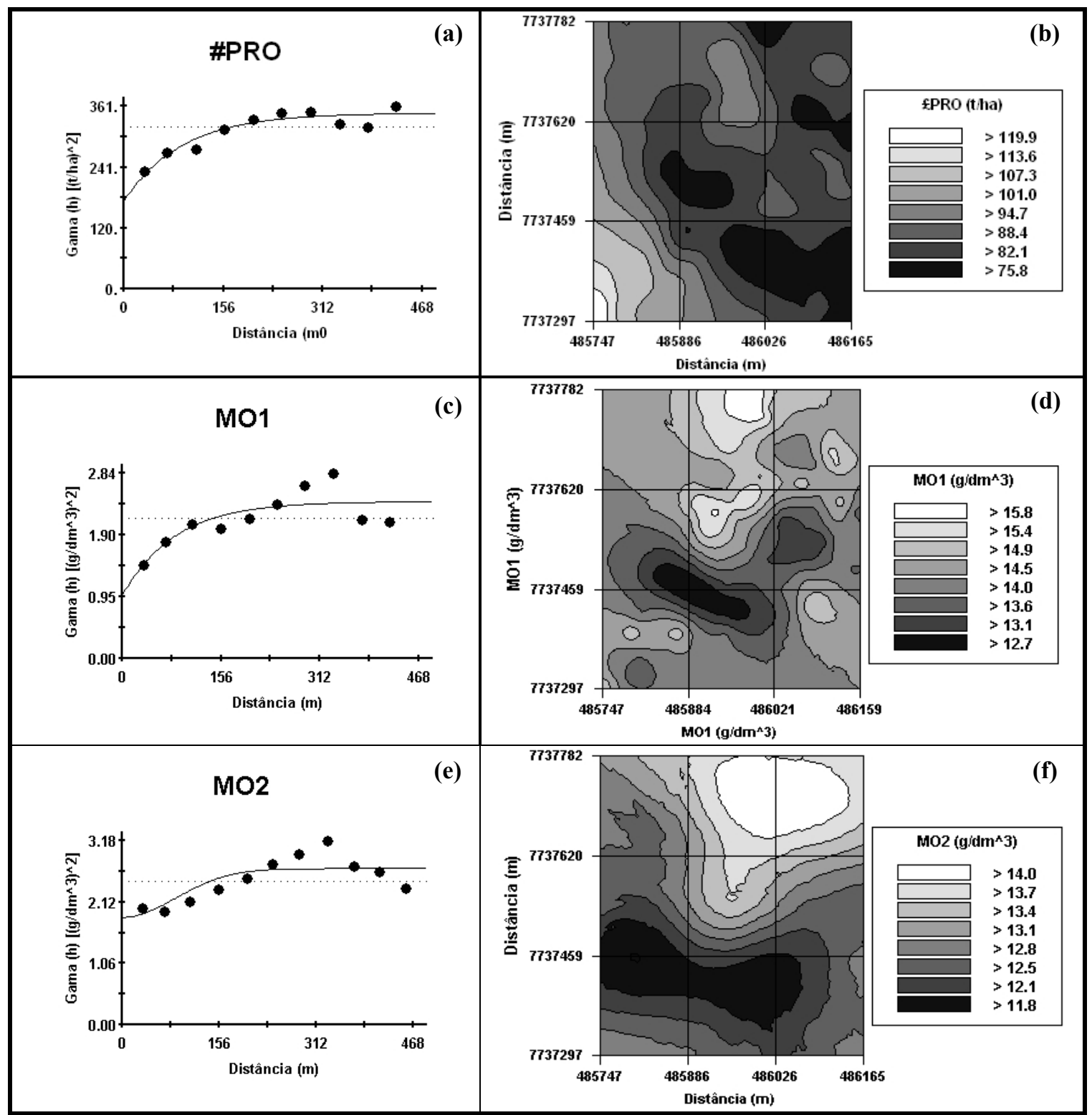

Fonte: Elaboração dos autores.

Nas Figuras 3 e 5, as cokrigagens diretas \#PRO$=\mathrm{f}(\mathrm{MO} 1)$ e $\# \mathrm{PRO}=\mathrm{f}(\mathrm{MO} 2)$ apresentaram, nas zonas de menores teores de MO1 (12,7-14,0 g $\left.\mathrm{dm}^{-3}\right)$, coincidentes com os menores teores de MO2 $\left(11,8-12,8 \mathrm{~g} \mathrm{dm}^{-3}\right)$, as menores £PRO $(75,8-94,7 \mathrm{t}$ $\left.\mathrm{ha}^{-1}\right)$, conforme a Figura 3. Em contrapartida, nas zonas de maiores teores de MO1 (14,5-15,8 $\mathrm{g} \mathrm{dm}^{-}$ ${ }^{3}$ ), coincidentes com os de maiores teores de MO2 $\left(13,1-14,0 \mathrm{~g} \mathrm{dm}^{-3}\right)$, as maiores $£$ PRO (101,0-119,9 t $\left.\mathrm{ha}^{-1}\right)$. Portanto, ambos os atributos (MO1 e MO2), por terem evidenciado apreciável relação espacial direta com a £PRO (Figura 5), podem ser perfeitamente utilizados como indicadores da £PRO, quando o objetivo for o de incrementar a produtividade de colmos de cana-de-açúcar. Tais resultados são análogos àqueles de Dalchiavon (2010) e 
Dalchiavon et al. (2011a), que correlacionaram função do teor de matéria orgânica do solo, assim espacial e diretamente a produtividade de arroz como Braga (2011), para a produtividade de colmos de terras altas e do feijoeiro, respectivamente, em de cana-de-açúcar em função também do teor de matéria orgânica do solo.

Figura 4. Semivariogramas simples de atributos químicos de um Argissolo Vermelho distrófico de Suzanápolis (SP) cultivado com cana-de-açúcar.

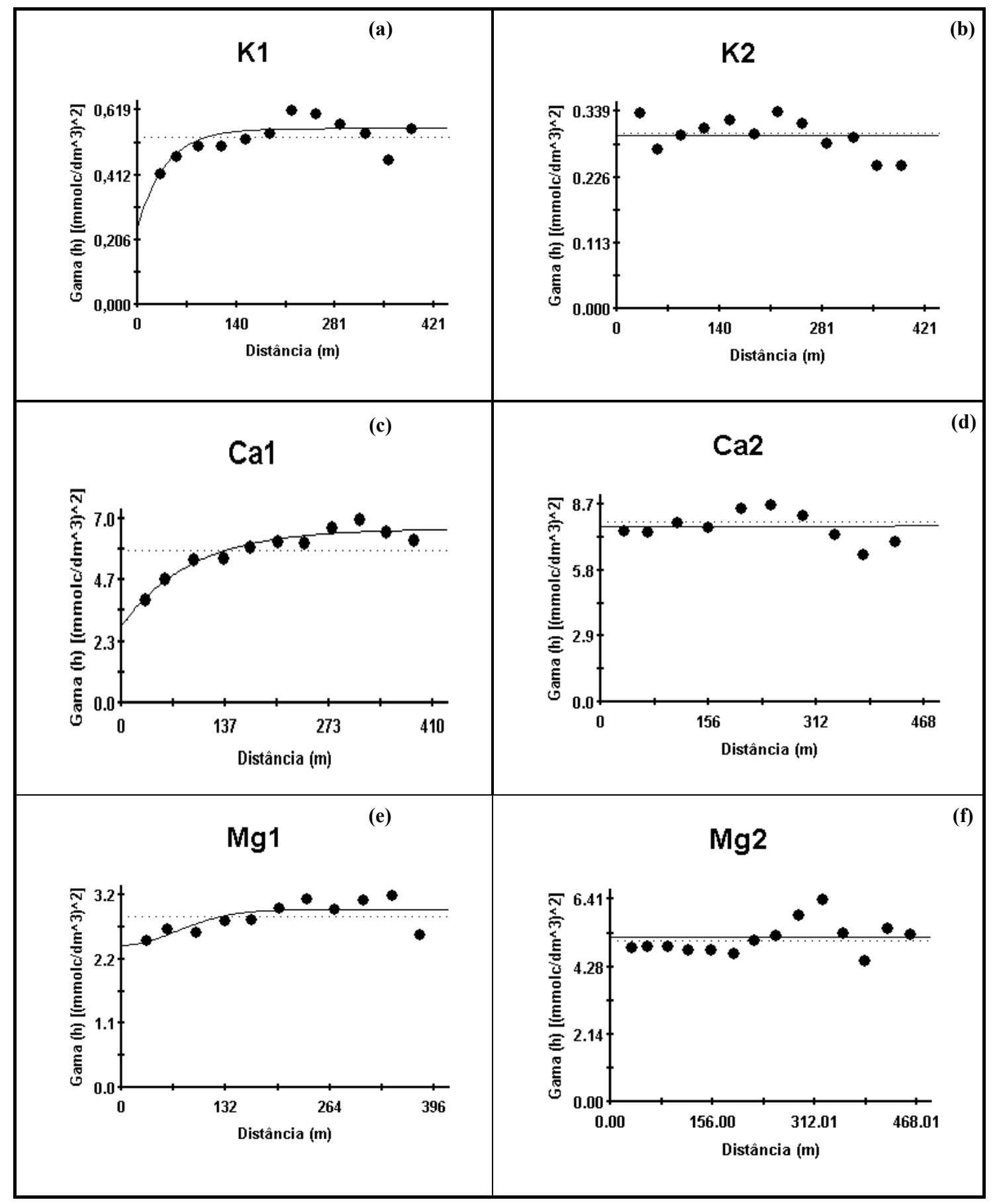

Fonte: Elaboração dos autores. 
Figura 5. Semivariogramas cruzados e mapas de cokrigagem da PRO em função da MO1 e da MO2 num Argissolo Vermelho distrófico de Suzanápolis (SP).

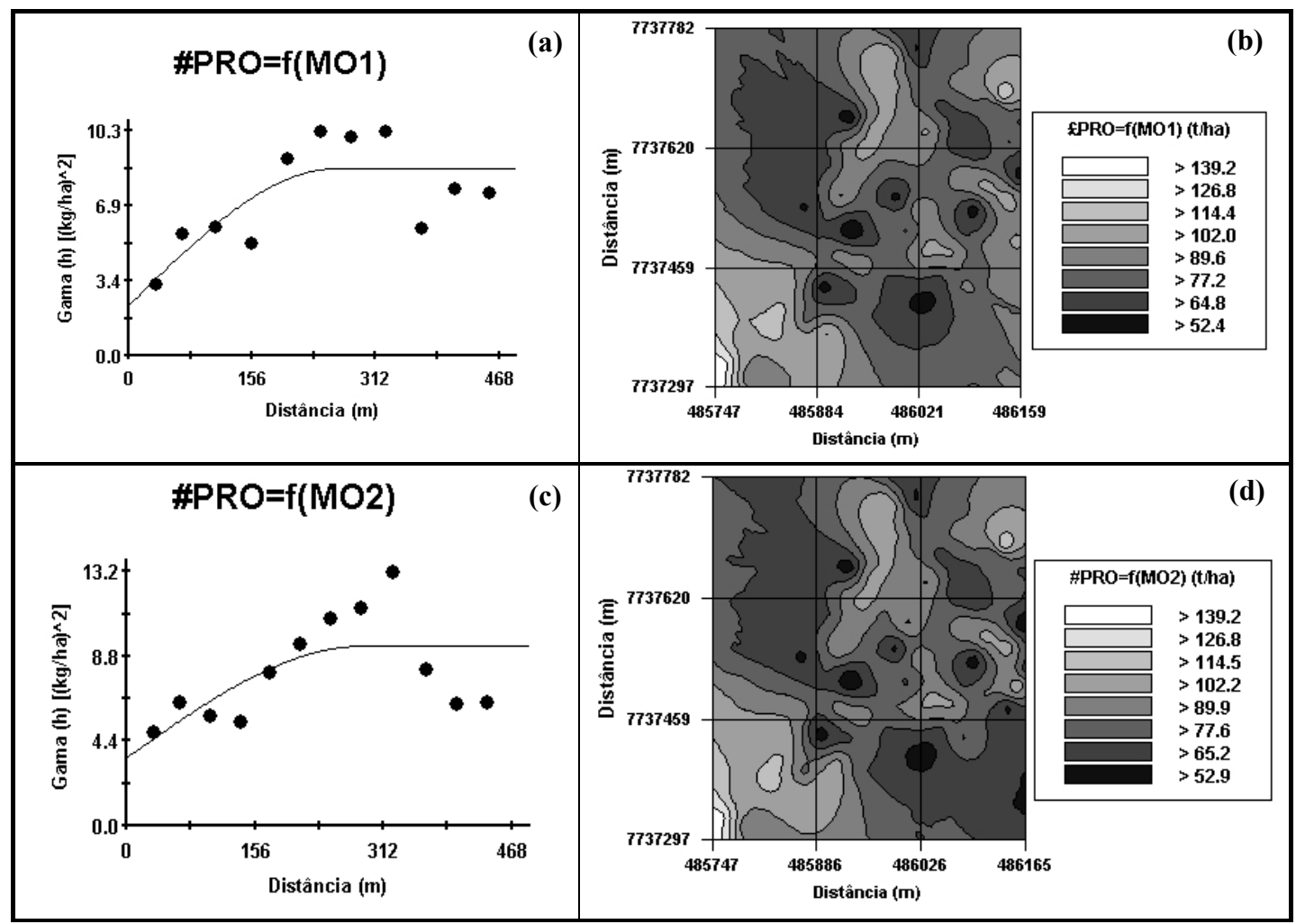

Fonte: Elaboração dos autores.

\section{Conclusão}

Os teores de matéria orgânica do solo, por evidenciarem correlações, Sperman's Rho e espaciais, com a produtividade de colmos de cana-de-açúcar, são indicadores de duas zonas específicas de manejo do solo fortemente associadas à produtividade da cana-de-açúcar. Nelas, tal produtividade varia entre 75,8-94,7 e 101,0-119,9 $\mathrm{t}$ ha-1 quando respectivamente os teores de matéria orgânica forem de 12,7-14,0 e 14,5-15,8 $\mathrm{g} \mathrm{dm}^{-3}(0$ $0,20 \mathrm{~m})$ e $11,8-12,8$ e $13,1-14,0 \mathrm{~g} \mathrm{dm}^{-3}(0,20-0,40$ $\mathrm{m})$.

\section{Referências}

BRAGA, J. A. Inter-relações da produtividade de cana-de-açúcar com atributos físico-químicos de um Argissolo Vermelho eutrófico do noroeste paulista. 2011. Dissertação (Mestrado em Agronomia) - Faculdade de Engenharia. Universidade Estadual Paulista, Ilha Solteira.
CARVALHO, M. P.; DALCHIAVON, F. C; GIOIA, M. T.; BRAGA, J. A. Inter-relações da resistência mecânica, umidade e do teor de matéria orgânica de um Argissolo Vermelho paulista sobre a produtividade da cana-deaçúcar. In: REUNIÃO BRASILEIRADE FERTILIDADE DO SOLO E NUTRIÇÃO DE PLANTAS, 29., 2010, Guarapari. Anais... Guarapari: Fertbio, 2010. p. 1-5.

CHAVES, L. H. G.; FARIAS, C. H. A. Variabilidade espacial do estoque de carbono nos Tabuleiros costeiros da Paraíba: Solo cultivado com cana-de-açúcar. Revista Brasileira de Ciências Agrárias, Recife, v. 3, n. 1, p. 2025, 2008.

COMPANHIA NACIONAL DE ABASTECIMENTO - CONAB. Acompanhamento da safra brasileira: canade-açúcar. Terceiro Levantamento, dezembro/2011. Brasília: CONAB, 2011.20 p.

CORÁ, J. E.; ARAUJO, A. V.; PEREIRA, G. T.; BERALDO, J. M. G. Variabilidade espacial de atributos do solo para adoção do sistema de agricultura de precisão na cultura de cana-de-açúcar. Revista Brasileira de Ciência do Solo, Viçosa, MG, v. 28, n. 6, p. 1013-1021, 2004. 
DALCHIAVON, F. C. Aspectos da produtividade do arroz de terras altas irrigado com atributos químicos do solo sob plantio direto. 2010. Dissertação (Mestrado em Agronomia) - Faculdade de Engenharia. Universidade Estadual Paulista, Ilha Solteira.

DALCHIAVON, F. C.; CARVALHO, M. P. Correlação linear e espacial dos componentes de produção e produtividade da soja. Semina: Ciências Agrárias, Londrina, v. 33, n. 2, p. 541-552, 2012.

DALCHIAVON, F. C.; CARVALHO, M. P.; ANDREOTTI, M.; MONTANARI, R. Variabilidade espacial de atributos da fertilidade de um Latossolo Vermelho Distroférrico sob Sistema Plantio Direto. Revista Ciência Agronômica, Fortaleza, v. 43, n. 3, p. 453-461, 2012.

DALCHIAVON, F. C.; CARVALHO, M. P.; FREDDI, O. S.; ANDREOTTI, M.; MONTANARI, R. Variabilidade espacial da produtividade do feijoeiro correlacionada com atributos químicos de um Latossolo Vermelho Distroférrico sob sistema de semeadura direta. Bragantia, Campinas, v. 70, n. 4, p. 1-9, 2011 a.

DALCHIAVON, F. C.; CARVALHO, M.P.; NOGUEIRA, D. C.; ROMANO, D.; ABRANTES, F. L.; ASSIS, J. T.; OLIVEIRA, M. S. Produtividade da soja e resistência mecânica à penetração do solo sob sistema plantio direto no cerrado brasileiro. Pesquisa Agropecuária Tropical, Goiás, v. 41, n. 1, p. 8-19, 2011 b.

DAVIS, J. C. Statistics and analysis in geology. 2. ed. New York: John Wiley, 1986. 646 p.

EMPRESA BRASILEIRA DE PESQUISA AGROPECUÁRIA - EMBRAPA. Centro Nacional de Pesquisas de Solos. Sistema brasileiro de classificação de solos. 2. ed. Rio de Janeiro, 2006. 412 p.

$G S^{+}$. Geostatistics for environmental sciences. 7. ed. Michigan: Plainwell, Gamma Design Software, 2004. $159 \mathrm{p}$.

LIMA, C. G. R.; CARVALHO, M. P.; NARIMATSU, K. C. P.; SILVA, M. G.; QUEIROZ, H. A. Atributos físicoquímicos de um Latossolo do cerrado brasileiro e sua relação com características dendrométricas do eucalipto. Revista Brasileira de Ciência do Solo, Viçosa, MG, v. 34, n. 1, p. 163-173, 2010.

MARTINS, M. V.; CARVALHO, M. P.; ANDREOTTI, M.; MONTANARI, R. Correlação linear e espacial entre a produtividade do feijoeiro e atributos físicos de um Latossolo Vermelho distroférrico de Selvíria, Estado de Mato Grosso do Sul. Acta Scientiarum Agronomy, Maringá, v. 31, n. 1, p. 147-154, 2009.
MOLIN, J. P.; VIEIRA JUNIOR, P. A.; DOURADO NETO, D.; FAULIN, G. C.; MASCARIN, L. Variação espacial na produtividade de milho safrinha devido aos macronutrientes e à população de plantas. Revista Brasileira de Milho e Sorgo, Sete Lagoas, v. 6, n. 3, p. 309-324, 2007.

MONTANARI, R.; CARVALHO, M. P.; ANDREOTTI, M.; DALCHIAVON, F. C.; LOVERA, L. H.; HONORATO, M. A. O. Aspectos da produtividade do feijão correlacionados com atributos físicos do solo sob elevado nível tecnológico de manejo. Revista Brasileira de Ciência do Solo, Viçosa, MG, v. 34, n. 6, p. 18111822, 2010.

MONTANARI, R.; PEREIRA, G. T.; MARQUES JÚNIOR, J.; SOUZA, Z. M.; PAZETO; J. R.; CAMARGO, L. A. L. Variabilidade espacial de atributos químicos em Latossolos e Argissolos. Ciência Rural, Santa Maria, v. 38, n. 5, p. 1266-1272, 2008.

RAIJ, B. V.; ANDRADE, J. C.; CANTARELlA, H.; QUAGGIO, J. A. Análise química para avaliação da fertilidade de solos tropicais. Campinas: Instituto Agronômico, 2001. 285 p.

SCHLOTZHAVER, S. D.; LITTLEL, R. C. SAS: system for elementary statistical analysis. 2. ed. Cary: SAS, 1997. $441 \mathrm{p}$.

SHAPIRO, S. S.; WILK, M. B. An analysys of variance test for normality: complete samples. Biometrika, London, v. 52, n. 3-4, p. 591-611, 1965.

SOUZA, Z. M.; CERRI, D. G. P.; COLET, M. J.; RODRIGUES, L. H. A.; MAGALHÃES, P. S. G.; MANDONI, R. J. A. Análise dos atributos do solo e da produtividade da cultura de cana-de-açúcar com o uso da geoestatística e árvore de decisão. Ciência Rural, Santa Maria, v. 40, n. 4, p. 840-847, 2010.

SOUZA, Z. M.; CERRI, P. D. G.; MAGALHÃES, P. G.; CAMPOS, M. C. C. Correlação dos atributos físicos e químicos do solo com a produtividade de cana-de-açúcar. Revista de Biologia e Ciências da Terra, João Pessoa, v. 8, n. 2, p. 183-190, 2008.

VITTI, A. C.; CANTARELLA, H.; TRIVELIN, P. C. O.; ROSSETTO, R. Nitrogênio. In: DINARDO-MIRANDA, L. L.; VASCONCELOS, A. C. M.; LANDELL, M. G. A. (Ed.). Cana-de-açúcar. Campinas: FunAg, 2008. p. 239-270.

WATANABE, R. T.; FIORETTO, R. A.; HERMANN, R. Propriedades químicas do solo e produtividade da canade-açúcar em função da adição da palhada de colheita, calcário e vinhaça em superfície (sem mobilização). Semina: Ciências Agrárias, Londrina, v. 25, n. 2, p. 93100, 2004. 